\title{
Very Low Disease Activity, DAPSA Remission, and Impact of Disease in a Spanish Population with Psoriatic Arthritis
}

\author{
Ruben Queiro (1), Juan D. Cañete, Carlos Montilla, Miguel Angel Abad, María Montoro, \\ Susana Gómez, and Ana Cábez, for the MAAPS Study Group
}

\begin{abstract}
Objective. To examine the grade of agreement between very low disease activity (VLDA) and Disease Activity Index for Psoriatic Arthritis (DAPSA) remission, as well as their association with the effect of the disease as assessed by the Psoriatic Arthritis Impact of Disease (PsAID) questionnaire in patients with psoriatic arthritis in routine clinical practice.

Methods. Posthoc analysis of data from a cross-sectional multicenter study. Patients were included who fulfilled the Classification for Psoriatic Arthritis (CASPAR) criteria with at least 1 year of disease duration and were treated with biological and/or conventional synthetic disease-modifying antirheumatic drugs according to routine clinical practice in Spain. Patients were considered in VLDA if they met $7 / 7$ of the minimal disease activity criteria. DAPSA and clinical (c)DAPSA score $\leq 4$ identified remissions.

Results. Of the 227 patients included in the original study, 26 (11.5\%), 52 (22.9\%), and 65 (28.6\%) were in VLDA, DAPSA remission, and cDAPSA remission, respectively. There was a moderate agreement between VLDA and DAPSA remission $(\kappa=0.52)$ or cDAPSA remission $(\kappa=0.42)$. Patients with VLDA had less effect of the disease as measured by PsAID [mean total score (SD): VLDA 1.1 (1.2); DAPSA remission 1.3 (1.5); cDAPSA remission 1.7 (1.6)]. There was a moderate agreement between DAPSA remission or cDAPSA remission and PsAID $<4(\kappa=0.46$ and $\kappa=0.58$ respectively), while poor agreement $(\kappa=0.18)$ was found between VLDA and PsAID $<4$.

Conclusion. VLDA criteria seem to be more stringent for assessing a status of remission; however, DAPSA remission shows better correlation with a patient-acceptable symptoms state than VLDA does. (First Release February 1 2019; J Rheumatol 2019;46:710-15; doi:10.3899/jrheum.180460)
\end{abstract}

\section{Key Indexing Terms:}

DISEASE ACTIVITY REMISSION PSORIATIC ARTHRITIS DISEASE ACTIVITY CRITERIA

Psoriatic arthritis (PsA) is a chronic inflammatory musculoskeletal disease, usually seronegative for rheumatoid factor and associated with psoriasis, with a prevalence of $0.02-0.42 \%{ }^{1}$ in the general population and $13.8-30 \%$ among patients with psoriasis ${ }^{1,2}$.

The target of therapy in PsA is to reach a state of remission

From the Department of Rheumatology, Hospital Universitario Central de Asturias, Oviedo; Department of Rheumatology, Hospital Universitario Clinic de Barcelona and Institut d'Investigacions Biomèdiques August Pi $i$ Sunyer (IDIBAPS), Barcelona; Department of Rheumatology, Hospital Universitario de Salamanca, Salamanca; Department of Rheumatology, Hospital Virgen del Puerto, Plasencia; Medical Department, Pfizer, Madrid, Spain.

This study was funded by Pfizer SLU.

R. Queiro, MD, PhD, Department of Rheumatology, Hospital Universitario Central de Asturias; J.D. Cañete, MD, PhD, Department of Rheumatology, Hospital Universitario Clinic de Barcelona and IDIBAPS; C. Montilla, MD, Department of Rheumatology, Hospital Universitario de Salamanca; M.A. Abad, MD, Department of Rheumatology, Hospital Virgen del Puerto; M. Montoro, MD, Medical Department, Pfizer; S. Gómez, MD, Medical Department, Pfizer; A. Cábez, MD, Medical Department, Pfizer.

Address correspondence to Dr.R. Queiro, Av. Roma, s/n, 33011 Oviedo, Asturias, Spain.E-mail: rubenque7@yahoo.es

Accepted for publication October 16, 2018. or at least minimal disease activity (MDA). Measuring disease activity has been a challenge owing to the diverse manifestations of the disease, including the skin and the musculoskeletal system ${ }^{3}$. Currently, different composite indices have been developed specifically for PsA, most focusing on multiple domains considered important to assess the disease activity states ${ }^{4}$. However, few data have been published on comparing these measures ${ }^{5}$.

In 2010, Coates, et $a l^{6}$ developed a composite outcome measure as a target of treatment for patients with PsA that encompasses skin, enthesitis, tender and swollen joint counts, and patient-reported domains [pain, the Health Assessment Questionnaire (HAQ), and global disease activity]. These criteria for MDA were validated using interventional trial data $^{7}$ and could be a reliable target for anti-tumor necrosis factor- $\alpha$ (anti-TNF- $\alpha$ ) therapy ${ }^{8,9,10}$. The same authors developed a more stringent definition of remission, in which all MDA 7/7 criteria (very low disease activity; VLDA) had to be satisfied ${ }^{3}$.

Previously, Schoels, et al ${ }^{11}$ proposed the remission criteria for the Disease Activity Index for Psoriatic Arthritis (DAPSA), which can be calculated with or without

Personal non-commercial use only. The Journal of Rheumatology Copyright ()$_{2019}$. All rights reserved. 
C-reactive protein [CRP; clinical DAPSA (cDAPSA)] and includes tender and swollen joint counts, and patient-reported pain and global disease activity scores.

It is well known that PsA has a considerable effect on patients' lives ${ }^{12}$. Patients with PsA experience significant disability and reduced quality of life, resulting from the emotional distress and functional impairment associated with psoriatic skin lesions, as well as arthritic joints ${ }^{10,13}$. Gossec, et al have developed and validated the PsA Impact of Disease (PsAID) questionnaire, which can be used to calculate a score reflecting the effect of PsA on patients' lives ${ }^{14}$. The PsAID questionnaire assessed 12 health and psychological domains, ranging from pain or physical activity, to anxiety or embarrassment. Scores lower than 4 identify a patient-acceptable symptoms state.

The aims of the present posthoc analysis were to examine the grade of agreement relationship between VLDA and DAPSA remission, as well as their association with the effect of the disease as assessed by the PsAID questionnaire in patients with PsA seen in routine clinical practice.

\section{MATERIALS AND METHODS}

This was a posthoc analysis of data from an observational, multicenter, crosssectional, and retrospective study carried out at 25 rheumatology outpatient clinics in the whole of Spain ${ }^{15}$.

The study included outpatients of both sexes over 18 years of age diagnosed with PsA according to the Classification for Psoriatic Arthritis (CASPAR) criteria $^{16}$, with at least 1 year of disease duration, with hand and foot radiological tests carried out during the 6 months prior to the study visit, and under treatment with biological and/or conventional synthetic disease-modifying antirheumatic drugs (csDMARD).

All patients provided their informed written consent to publish the material. In accordance with the Spanish recommendations, the study was approved by the Clinical Research Ethics Committee of La Fe Hospital [ref number: FPNT-07-14-EO (C)] and was conducted in accordance with the principles contained in the Declaration of Helsinki for studies in humans.

Data were collected between May 2014 and February 2015 at the single study visit. Patient data collection included demographics and clinical characteristics ${ }^{15}$. Patients completed the self-reported PsAID questionnaire ${ }^{14}$.

Patients were considered in VLDA when they met all MDA 7/7 criteria proposed by Coates, et $\mathrm{l}^{3,6}$ : tender joint count $\leq 1$; swollen joint count $\leq 1$; Psoriasis Area and Severity Index score $\leq 1$ or body surface area $\leq 3 \%$; patient pain visual analog scale (VAS) score $\leq 15$; patient global disease activity VAS score $\leq 20$; HAQ score $\leq 0.5$; and tender entheseal points $\leq 1$.

DAPSA remission was defined according to Schoels, et al ${ }^{11,17}$. DAPSA score was calculated by adding the number of tender and swollen joints, VAS pain, patient's global assessment (PtGA), and CRP (mg/dl). The cDAPSA was calculated without the contribution of CRP. DAPSA and cDAPSA score $\leq 4$ identified remissions ${ }^{11}$.

The PsAID questionnaire 14 reflects the impact of PsA from the patient's perspective. It is composed of 12 physical and psychological domains. Each domain is rated from 0 to 10 with a different weighting. Total score is divided by 20 . The final score has a range from 0 (best status) to 10 (worst status) with a cutoff of 4 . PsAID score $<4$ identified patient-acceptable symptoms state.

Statistical methodology. A descriptive statistical analysis of all the variables was performed, including central tendency and dispersion measures for continuous variables, and absolute and relative frequencies for categorical variables. Student t test, Mann-Whitney U test, or Kruskall-Wallis H test were used to compare quantitative variables, and Pearson chi-squared or Fisher's exact tests for qualitative variables. Concordance was assessed using Cohen $\kappa$ and was considered as follows: $<0.20=$ poor, $0.21-0.40=$ fair,
$0.41-0.60=$ moderate, $0.61-0.80=$ good , and $0.81-1.00=$ very good Tests were 2-tailed with a significance level of $5 \%$. Data were analyzed using SPSS v19.0 statistical software.

\section{RESULTS}

Of the 227 patients included, 26 (11.5\%), 52 (22.9\%), and 65 (28.6\%) were in VLDA, DAPSA remission, and cDAPSA remission, respectively. Patient demographics and clinical information are given in Table 1. Most of the patients were men (65.4-67.7\%) with a mean age from 52.0 to 53.7 years, and mostly had peripheral arthritis (84.6-87.7\%). The mean CRP level ranged from $1.9 \mathrm{mg} / \mathrm{l}$ to $2.2 \mathrm{mg} / \mathrm{l}$. Familial history of psoriasis was present in 53.8-55.4\% of the patients. PsA disease duration was 8.9-9.5 years (Table 1).

PsA status at study visit is shown in Table 1. Erosive disease in hands and/or feet was detected in 50.0\%, 43.8\%, and $41.5 \%$ of VLDA, DAPSA $\leq 4$, and cDAPSA $\leq 4$ patients, respectively. At the study visit, anti-TNF treatment in monotherapy was most frequent in patients with VLDA (42.3\% VLDA vs $29.2 \%$ DAPSA $\leq 4$ and $26.2 \%$ DAPSA $\leq 4$; Table 2). Conversely, $34.6 \%$ of VLDA patients received csDMARD monotherapy compared with $41.7 \%$ and $44.6 \%$ of DAPSA remission and cDAPSA remission, respectively (Table 2).

Table 3 examines the relationship between VLDA and the other indices of remission. There was a moderate agreement between VLDA and DAPSA remission $(\kappa=0.52)$ or cDAPSA remission $(\kappa=0.42)$.

Patients with VLDA had less impact of the disease as measured by PsAID [mean total score (SD): VLDA 1.1 (1.2); DAPSA remission 1.3 (1.5); cDAPSA remission 1.7 (1.6); Table 1]. Twenty-five (96.2\%) patients with VLDA, 42/52 $(80.8 \%)$ in DAPSA remission, and $57(89.1 \%)$ in cDAPSA remission obtained a PsAID score $<4$. There was moderate agreement between DAPSA or cDAPSA and PsAID $<4$ $(\kappa=0.55$ and $\kappa=0.58$, respectively), while poor agreement ( $\kappa=0.18)$ was found between VLDA and PsAID < 4; Table $3)$. Figure 1 examines PsAID domains score according to VLDA state, DAPSA remission, and cDAPSA remission. Overall, patients who were in DAPSA remission, and in particular cDAPSA remission, obtained a higher score in all PsAID domains than did patients with VLDA. Only 2 physical domains, fatigue and pain, had a deeper effect on patients with VLDA than on those in DAPSA remission. Statistically significant differences were observed between patients who were in DAPSA and cDAPSA remission or in a VLDA state and those who were not for all PsAID domains ( $p<0.001$, data not shown).

\section{DISCUSSION}

Remission or low disease activity is the goal of therapy in PsA. In recent years, the development of composite indices tailored for the assessment of PsA disease activity led to the question of which is the best tool for identifying a state of real remission ${ }^{18}$. 
Table 1. Demographic and clinical characteristics of the study population.

\begin{tabular}{|c|c|c|c|}
\hline Characteristics & $\begin{array}{l}\text { VLDA, } \\
\mathrm{n}=26\end{array}$ & $\begin{array}{c}\text { DAPSA } \leq 4 \\
\mathrm{n}=52^{*}\end{array}$ & $\begin{array}{c}\text { cDAPSA } \leq 4 \\
\mathrm{n}=65\end{array}$ \\
\hline Male, n (\%) & $17(65.4)$ & $32(66.7)$ & $44(67.7)$ \\
\hline Age, yrs, mean (SD) & $53.7(14.4)$ & $52.0(13.5)$ & $52.1(13.3)$ \\
\hline CRP, mg/l, mean (SD) & $1.9(1.9)$ & $1.9(2.0)$ & $2.2(2.6)$ \\
\hline \multicolumn{4}{|l|}{ Comorbidities, $\mathrm{n}(\%)$} \\
\hline Dyslipidemia & $11(42.3)$ & $21(43.8)$ & $24(36.9)$ \\
\hline HBP & $9(34.6)$ & $11(22.9)$ & $15(23.1)$ \\
\hline Obesity & $9(34.6)$ & $15(31.3)$ & $22(33.8)$ \\
\hline DM & $3(11.5)$ & $4(8.3)$ & $7(10.8)$ \\
\hline \multicolumn{4}{|l|}{ PsA characteristics } \\
\hline \multicolumn{4}{|l|}{ PsA clinical pattern, n (\%) } \\
\hline Axial & $0(0.0)$ & $1(2.1)$ & $2(3.1)$ \\
\hline Peripheral & $22(84.6)$ & $42(87.5)$ & $57(87.7)$ \\
\hline Mixed & $4(15.4)$ & $5(10.4)$ & $6(9.2)$ \\
\hline Dactylitis, n (\%) & $13(50.0)$ & $22(45.8)$ & $31(47.7)$ \\
\hline Enthesitis, n (\%) & $13(50.0)$ & $17(35.4)$ & $26(40.0)$ \\
\hline \multicolumn{4}{|l|}{ Familial history, n (\%) } \\
\hline Psoriasis & $14(53.8)$ & $26(54.2)$ & $36(55.4)$ \\
\hline PsA & $0(0.0)$ & $4(8.3)$ & $5(7.7)$ \\
\hline Ankylosing spondylitis & $1(3.8)$ & $1(2.1)$ & $1(1.5)$ \\
\hline PsA duration, yrs, mean (SD) & $9.5(9.1)$ & $9.4(7.5)$ & $8.9(6.8)$ \\
\hline Skin symptoms duration, yrs, mean (SD) & $22.0(16.7)$ & $19.3(15.4)$ & $19.2(14.7)$ \\
\hline Articular symptoms duration, yrs, mean (SD) & $12.0(9.8)$ & $11.5(8.8)$ & $11.2(8.5)$ \\
\hline \multicolumn{4}{|l|}{ PsA status at study visit } \\
\hline \multicolumn{4}{|l|}{ Radiologic findings } \\
\hline Erosions in hands and/or feet, $\mathrm{n}(\%)$ & $13(50.0)$ & $21(43.8)$ & $27(41.5)$ \\
\hline PsAID, mean (SD) & $1.1(1.2)$ & $1.3(1.5)$ & $1.7(1.6)$ \\
\hline PASI, mean (SD) & $0.4(0.8)$ & $0.6(1.1)$ & $0.7(1.4)$ \\
\hline HAQ, mean (SD) & $0.06(0.1)$ & $0.1(0.3)$ & $0.2(0.3)$ \\
\hline DAPSA, mean (SD) & $1.5(1.6)$ & $1.6(1.1)$ & $2.2(1.4)$ \\
\hline cDAPSA, mean (SD) & $1.3(1.6)$ & $1.5(1.0)$ & $2.0(1.4)$ \\
\hline
\end{tabular}

* There were 52 patients in DAPSA remission, but complete information was available for only 48. VLDA: very low disease activity; CRP: C-reactive protein; HBP: high blood pressure; DM: diabetes mellitus; PASI: Psoriasis Area and Severity Index; HAQ: Health Assessment Questionnaire; PsAID: Psoriatic Arthritis Impact of Disease; DAPSA: Disease Activity Index for Psoriatic Arthritis; cDAPSA: clinical DAPSA; PsA: psoriatic arthritis.

Table 2. PsA treatment at study visit.

\begin{tabular}{lccc}
\hline Treatments & $\begin{array}{c}\text { VLDA, } \\
\mathrm{n}=26\end{array}$ & $\begin{array}{c}\text { DAPSA } \leq 4, \\
\mathrm{n}=52^{\wedge}\end{array}$ & $\begin{array}{c}\text { cDAPSA } \leq 4, \\
\mathrm{n}=65\end{array}$ \\
\hline $\begin{array}{l}\text { Treatment pattern } \\
\quad \text { Anti-TNF monotherapy } \\
\text { csDMARD and anti-TNF } \\
\quad \text { csDMARD monotherapy }\end{array}$ & $11(42.3)$ & $14(29.2)$ & $17(26.2)$ \\
Anti-TNF- $\alpha^{*}$ & $6(23.1)$ & $14(29.2)$ & $19(29.2)$ \\
$\quad$ Etanercept & $9(34.6)$ & $20(41.7)$ & $29(44.6)$ \\
$\quad$ Adalimumab & $8(47.1)$ & $12(42.9)$ & $16(44.4)$ \\
$\quad$ Infliximab & $8(47.1)$ & $12(42.9)$ & $15(41.7)$ \\
$\quad$ Golimumab & $0(0.0)$ & $3(10.7)$ & $3(8.3)$ \\
NSAID** & $1(5.9)$ & $1(3.6)$ & $1(2.8)$ \\
Infiltrations** & $9(34.6)$ & $19(39.6)$ & $28(43.1)$ \\
Corticoids** & $2(7.7)$ & $11(22.9)$ & $14(21.5)$ \\
& $1(3.8)$ & $5(10.4)$ & $7(10.8)$ \\
\hline
\end{tabular}

Values are $\mathrm{n}(\%){ }^{\wedge}$ There were 52 patients in DAPSA remission, but complete information was available for only 48. * Monotherapy or combination therapy; ** Combination therapy. PsA: psoriatic arthritis; VLDA: very low disease activity; DAPSA: Disease Activity Index for Psoriatic Arthritis; cDAPSA: clinical DAPSA; NSAID: nonsteroidal antiinflammatory drugs; csDMARD: conventional synthetic disease-modifying antirheumatic drugs; TNF: tumor necrosis factor. 
Table 3. Concordance between VLDA and other indices of remission and PsAID.

\begin{tabular}{lcc}
\hline Variables & $\kappa$ & $95 \%$ CI \\
\hline DAPSA $\leq 4$ vs VLDA & 0.52 & $(0.38-0.67)$ \\
cDAPSA $\leq 4$ vs VLDA & 0.42 & $(0.29-0.55)$ \\
DAPSA $\leq 4$ vs PsAID $<4$ & 0.46 & $(0.33-0.57)$ \\
cDAPSA $\leq 4$ vs PsAID $<4$ & 0.58 & $(0.47-0.70)$ \\
VLDA vs PsAID $<4$ & 0.18 & $(0.10-0.25)$ \\
\hline
\end{tabular}

VLDA: very low disease activity; DAPSA: Disease Activity Index for Psoriatic Arthritis; cDAPSA: clinical DAPSA; PsAID: Psoriatic Arthritis Impact of Disease questionnaire.

For patients to be in MDA 5/7 is not the same as being in remission, because they may have some residual disease activity $^{3}$. Studies aimed at comparing the composite indices tailored for PsA showed that VLDA seems to be reliable in assessing a condition of disease remission ${ }^{3,6,18}$. Data from our present study seem to be in concordance with published data that show that VLDA criteria are among the most stringent for assessing a status of remission ${ }^{5,18}$. In our study the proportion of patients receiving anti-TNF and/or csDMARD therapy who were in VLDA state $(11.5 \%)$ was lower than the proportion of those considered in remission state as measured by DAPSA (22.9\%) or cDAPSA (28.6\%). Recently, a cross-sectional study carried out with 109 patients reported a prevalence of $17.4 \%, 21.1 \%$, and $25.6 \%$ of patients with VLDA, DAPSA remission, and cDAPSA remission state, respectively ${ }^{18}$. These outcomes were achieved at a higher rate in patients receiving anti-TNF therapy compared with csDMARD ${ }^{18}$. Lubrano, et al showed a similar rate of patients achieving VLDA criteria and DAPSA remission (37.3\% and $36 \%$, respectively) after 12 months of anti-TNF therapy ${ }^{19}$.

Similar to a previous study ${ }^{19}$, our results have shown that there is only a moderate relationship between VLDA and DAPSA remission. Although DAPSA is a good instrument for assessing low disease status, some authors have pointed out that its potential weakness is that it is an instrument for evaluating peripheral arthritis only, with a risk of underestimating disease activity in such important domains as skin, axial disease, and enthesitis ${ }^{18}$. However, although DAPSA includes only joint counts as musculoskeletal manifestations of PsA, it also includes patient pain assessment and PtGA, which might record such other domains as enthesitis and axial disease ${ }^{20}$.

PsA is a multifaceted chronic inflammatory disease with different manifestations and implications. Patients with PsA experience functional impairment and reduced quality of life, so PtGA in PsA is explained mainly by the physical, but also by the psychological, aspect of the disease ${ }^{21}$. Patient-reported outcomes, such as the novel PsAID ${ }^{14}$ questionnaire, are important instruments for evaluating healthcare interventions and for reflecting the effect of PsA on patients' lives. There are few studies that have reported results with this tool ${ }^{15,21,22}$.

Recently, we have shown statistically significant differences between MDA and non-MDA patients in all physical and psychological PsAID domains ${ }^{15}$. As expected, in the present analysis a significantly lesser effect of the disease was also observed for all PsAID domains in patients who were in

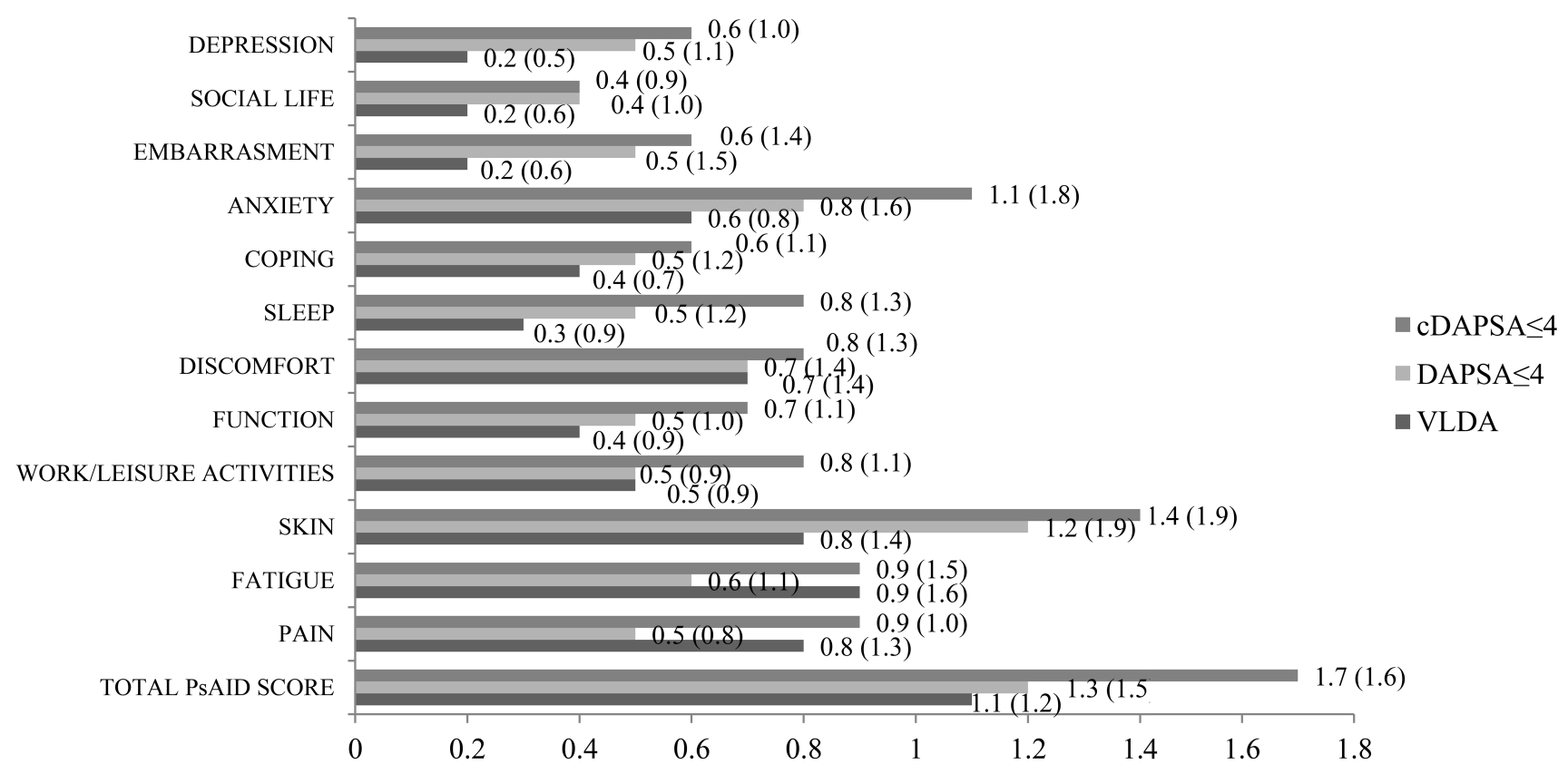

Figure 1. PsAID score according to VLDA state, DAPSA remission, and cDAPSA remission. PsAID: Psoriatic Arthritis Impact of Disease; VLDA: very low disease activity; DAPSA: Disease Activity Index for Psoriatic Arthritis; cDAPSA: clinical DAPSA.

Personal non-commercial use only. The Journal of Rheumatology Copyright (C) 2019. All rights reserved. 
DAPSA remission or in a VLDA state, compared to those who were not.

Our study shows that patients with VLDA seem have a lower effect of the disease as measured by PsAID as a whole, compared with those in DAPSA remission. In fact, the proportion of patients with a PsAID score $<4$ was higher in the VLDA group than in the DAPSA remission groups. However, curiously enough, while poor agreement $(\kappa=0.18)$ was found between VLDA and PsAID $<4$, it was moderate between DAPSA or cDAPSA and PsAID $<4(\kappa=0.46$ and $\kappa=0.58$, respectively). This could explain why fatigue, discomfort, and pain, 3 of the domains predominantly related to physical effect due to joint damage ${ }^{21}$, achieved the highest scores in patients with VLDA. Skin lesions have a considerable effect on quality of life, particularly in physiological symptoms, but can heal without scarring, while inflammation of the joints has cumulative effects, potentially leading to permanent destruction and impairment of physical function ${ }^{17}$. Therefore, in this cohort, these symptoms of the disease could carry more weight in the quality of life of the patient. Although DAPSA constitutes an instrument developed primarily to reflect the arthritis aspects of PsA, it correlates very well with functional status and radiological progression of PsA ${ }^{20}$.

Some limitations derived from the cross-sectional features of this study should be borne in mind. PsA is known to be a heterogeneous disorder, with a highly variable progression between individuals. The data presented here describe a specific moment of the state of illness of patients with a long evolution of the disease.

In our study we report that in normal clinical practice, around $11 \%$ and $30 \%$ of patients with PsA achieve a VLDA or DAPSA remission state, respectively, which are associated with a low effect of disease (PsAID < 4). Cutaneous and musculoskeletal symptoms could exert different influences on the different PsAID items. However, our results do not allow us to assert a greater weight of the musculoskeletal symptoms in the total PsAID, because the cutaneous involvement in this cohort was very mild. Although moderate agreement exists between VLDA and DAPSA, VLDA criteria seem to be more stringent for assessing a status of remission than are DAPSA $\leq 4$. However, DAPSA $\leq 4$ seems to reflect better the patients' point of view of disease.

\section{ACKNOWLEDGMENT}

The authors are grateful to Luz Samaniego for her valuable contribution in statistical analyses. We are also grateful to Esther Tapia for helping us with the medical writing of this manuscript.

\section{REFERENCES}

1. Chandran V, Raychaudhuri SP. Geoepidemiology and environmental factors of psoriasis and psoriatic arthritis. J Autoimmun 2010;34:J314-21.

2. Ibrahim G, Waxman R, Helliwell PS. The prevalence of psoriatic arthritis in people with psoriasis. Arthritis Rheum 2009;61:1373-8.

3. Coates LC, Helliwell PS. Defining low disease activity states in psoriatic arthritis using novel composite disease instruments. J Rheumatol 2016;43:371-5.

4. Lubrano E, Perrotta FM, Kavanaugh A. An overview of low disease activity and remission in psoriatic arthritis. Clin Exp Rheumatol 2015;33:S51-4.

5. van Mens LJ, van de Sande MG, van Kuijk AW, Baeten D, Coates LC. Ideal target for psoriatic arthritis? Comparison of remission and low disease activity states in a real-life cohort. Ann Rheum Dis 2018;77:251-7.

6. Coates LC, Fransen J, Helliwell PS. Defining minimal disease activity in psoriatic arthritis: a proposed objective target for treatment. Ann Rheum Dis 2010;69:48-53.

7. Coates LC, Helliwell PS. Validation of minimal disease activity criteria for psoriatic arthritis using interventional trial data. Arthritis Care Res 2010;62:965-9.

8. Perrotta FM, Marchesoni A, Lubrano E. Minimal disease activity and remission in psoriatic arthritis patients treated with anti-TNF- $\alpha$ drugs. J Rheumatol 2016;43:350-5.

9. Perrotta FM, Lubrano E. Subcutaneous anti-TNF alfa induced sustained minimal disease activity and remission in psoriatic arthritis patients: a retrospective study. Postgrad Med 2016;128:693-6.

10. Haddad A, Thavaneswaran A, Ruiz-Arruza I, Pellett F, Chandran V, Cook RJ, et al. Minimal disease activity and anti-tumor necrosis factor therapy in psoriatic arthritis. Arthritis Care Res 2015; 67:842-7.

11. Schoels MM, Aletaha D, Alasti F, Smolen JS. Disease activity in psoriatic arthritis (PsA): defining remission and treatment success using the DAPSA score. Ann Rheum Dis 2016;75:811-8.

12. Strand V, Sharp V, Koenig AS, Park G, Shi Y, Wang B, et al. Comparison of health-related quality of life in rheumatoid arthritis, psoriatic arthritis and psoriasis and effects of etanercept treatment. Ann Rheum Dis 2012;71:1143-50.

13. Borman P, Toy GG, Babaoglu S, Bodur H, Ciliz D, Alli N. A comparative evaluation of quality of life and life satisfaction in patients with psoriatic and rheumatoid arthritis. Clin Rheumatol 2007;26:330-4.

14. Gossec L, de Wit M, Kiltz U, Braun J, Kalyoncu U, Scrivo R, et al; EULAR PsAID Taskforce. A patient-derived and patient-reported outcome measure for assessing psoriatic arthritis: elaboration and preliminary validation of the Psoriatic Arthritis Impact of Disease (PsAID) questionnaire, a 13-country EULAR initiative. Ann Rheum Dis 2014;73:1012-9.

15. Queiro R, Cañete JD, Montilla C, Abad M, Montoro M, Gomez S, et al. Minimal disease activity and impact of disease in psoriatic arthritis: a Spanish cross-sectional multicenter study. Arthritis Res Ther 2017;19:72.

16. Taylor W, Gladman D, Helliwell P, Marchesoni A, Mease P, Mielants H; CASPAR Study Group. Classification criteria for psoriatic arthritis: development of new criteria from a large international study. Arthritis Rheum 2006;54:2665-73.

17. Smolen JS, Schoels M, Aletaha D. Disease activity and response assessment in psoriatic arthritis using the Disease Activity index for PSoriatic Arthritis (DAPSA). A brief review. Clin Exp Rheumatol 2015;5 Suppl 93:S48-50.

18. Lubrano E, De Socio A, Perrotta FM. Comparison of composite indices tailored for psoriatic arthritis treated with csDMARD and bDMARD: A cross-sectional analysis of a longitudinal cohort. J Rheumatol 2017;44:1159-64.

19. Lubrano E, Perrotta FM. Defining low disease activity states in psoriatic arthritis using novel composite disease instruments. J Rheumatol 2016;43:1765-6.

20. Aletaha D, Alasti F, Smolen JS. Disease activity states of the DAPSA, a psoriatic arthritis specific instrument, are valid against functional status and structural progression. Ann Rheum Dis 2017;76:418-21 
21. Talli S, Etcheto A, Fautrel B, Balanescu A, Braun J, Canete JD, et al. Patient global assessment in psoriatic arthritis - what does it mean? An analysis of 223 patients from the Psoriatic Arthritis Impact of Disease (PsAID) study. Joint Bone Spine 2016;83:335-40.

22. Salaffi F, Di Carlo M, Carotti M, Farah S, Gutierrez M. The Psoriatic Arthritis Impact of Disease 12-item questionnaire: equivalence, reliability, validity, and feasibility of the touch-screen administration versus the paper-and-pencil version. Ther Clin Risk Manag 2016;12:631-42.

\section{APPENDIX.}

List of study collaborators. MAAPS (Minimal Activity in Psoriatic Arthritis) Study Group: J.C. Torre Alonso (H. Monte Naranco, Oviedo, Spain); J.A. Román-Ivorra [Hospital Universitario (HU) La Fe, Valencia, Spain]; J. Sanz (HU Puerta de Hierro, Madrid, Spain); J. Salvatierra (HU San Cecilio, Granada, Spain); J. Calvo-Alén (HU Sierrallana, Torrelavega, Spain); A. Sellas (Vall d'Hebron, Barcelona, Spain); F.J. Rodriguez (Santa Lucia, Cartagena, Spain); A. Bermúdez (Virgen de la Arrixaca, Murcia, Spain); M. Romero (Complejo hospitalario Jaén, Spain); M. Riesco (Rheumatology H. Juan Ramón Jiménez, Huelva, Spain); J.C. Cobeta (H. Royo Villanova, Zaragoza, Spain); F. Medina (H. Puerta del Mar, Cádiz, Spain); A. Aragón (H. Getafe, Madrid, Spain); M.L. García (HU Basurto, Bilbao, Spain); A. Urruticoechea (H. Can Misses, Ibiza, Spain); C.M. González (HU Gregorio Marañón, Madrid, Spain); E. Judez (HU, Albacete, Spain); B. González (HU Nta. Sra de la Candelaria, Tenerife, Spain); P. Fernández (HU 12 de Octubre, Madrid, Spain); L. Pantoja (H. del Bierzo, Leon, Spain); R. Morlá (H. Sant Pau y Sta. Tecla, Tarragona, Spain). 


\section{Correction}

Very Low Disease Activity, DAPSA Remission, and Impact of Disease in a Spanish Population with Psoriatic Arthritis

Queiro R, Cañete JD, Montilla C, Abad MA, Montoro M, Gómez S, Cábez A, for the MAAPS Study Group. Very low disease activity, DAPSA remission, and impact of disease in a Spanish population with psoriatic arthritis. J Rheumatol 2019;46:710-15. In the Results section of the text, second paragraph, third sentence, the $26.2 \%$ should be cDAPSA.

doi:10.3899/jrheum.180460.C1 